\title{
An analysis of 7836 successive new outpatient referrals
}

\author{
G D PERKIN
}

From the Charing Cross Hospital, London, UK

SUMMARY An analysis has been performed of 7836 successive new outpatient referrals seen by one consultant neurologist. In approximately a quarter of the patients, a specific diagnosis was not possible. A further $30 \%$ of the total was accounted for by epilepsy, tension headache, cerebrovascular disease and migraine.

In a previous paper, 'I analysed the diagnoses of 4000 successive new outpatient referrals seen personally at Charing Cross and Hillingdon Hospitals. By the end of 1987, the number of cases available for analysis had risen to 7836 and these form the basis of the present communication.

\section{Patients and methods}

The diagnostic information on all new outpatient referrals seen personally between 1977 and 1987 has been analysed. Up to 1983, patients seen at Charing Cross Hospital represented $57.2 \%$ of the total, but in the last 5 years, the number of patients seen at the two hospitals has been almost equal (1970 and 1866). The cases seen personally account for approximately three-quarters of all the new referrals to my clinics. Only a single diagnosis has been allotted to each patient. If the patient had more than one diagnosis (for example, chronic tension headache together with occasional attacks of classical migraine) the predominant complaint has been used in the survey. Where the patient's presentation was the reflection of a particular neurological pathology (for example, a seizure secondary to a meningioma) classification is under the specific condition.

Clinical characteristics were used for many diagnoses, including epilepsy, cerebrovascular disease, tension and migraine headaches and the various extra-pyramidal disorders. Vahlquist's criteria ${ }^{2}$ have been used for the diagnosis of migraine and criteria proposed by the author ${ }^{1}$ for the diagnosis of tension headache. Cervical and lumbar spondylosis were diagnosed on the basis of an appropriate history accompanied by signs, whether motor, sensory or reflex, indicating a focal radiculopathy. Radiological evidence of cord compression was required when diagnosing cervical or thoracic myelopathy secondary to degenerative disease of the spinal column. Virtually every patient with an intracranial tumour had had the diagnosis confirmed histologically. Electrophysiological data were important,

Address for reprint requests: Dr G D Perkin, Charing Cross Hospital, Fulham Palace Road, London W6 8RF, UK.

Received 17 December 1988.

Accepted 21 December 1988 and sometimes essential, when diagnosing peripheral nerve disorders. All patients with a peripheral neuropathy had electrophysiological evidence of altered conduction, either sensory, motor or both in at least two peripheral nerves. Abnormal distal motor or sensory conduction in the median nerve was a pre-requisite for the diagnosis of carpal tunnel syndrome. Some of the other entrapment neuropathies (this category includes mononeuropathies triggered by abnormal angulation or external compression) were diagnosed clinically but, in the majority, support was forthcoming from electrophysiological criteria which were also used, in conjunction with biopsy findings, for establishing a diagnosis of primary muscle disease. For multiple sclerosis, the $\mathrm{MRC}^{3}$ classification has been used, with the exception that cases of isolated optic neuritis have been included.

The anatomical category includes patients in whom the physical signs allowed accurate anatomical localisation without revealing its pathological basis. Patients whose symptoms were due to a previously unrecognised disorder of another system, for example thyrotoxicosis, were classified separately. Approximately a quarter of the patients were left without a diagnosis. Many of them were followed, always personally, for a sufficient time to ensure that, as far as possible, a specific condition had not been overlooked.

The diagnosis of hyperventilation syndrome was accepted if the patient's symptoms could be reproduced by overbreathing, and there was no evidence of structural disease of the nervous system. In the patients with conversion hysteria, physical signs were demonstrated which, by their nature, necessitated elaboration. Some of these patients had evidence of co-existing organic disease but approximately half had the characteristic features of Briquet's syndrome. ${ }^{4}$

\section{Results}

Table 1 summarises the number of patients in the four major categories, comparing the distribution of the first 4000 cases, and the subsequent 3836 . Table 2 lists the twenty most common diagnoses, representing $88.5 \%$ of the total, to which the first five entries contribute nearly $57 \%$. A breakdown of all the cases is available from the author. In a previous publication, 'I assessed during two separate two-year periods the 
Table 1 Distribution of cases among four diagnostic categories

\begin{tabular}{lrrrr}
\hline & \multicolumn{2}{l}{$1977-1983$} & \multicolumn{2}{l}{$1983-1987$} \\
\hline Diagnosis & \multicolumn{1}{l}{ No. } & \multicolumn{1}{l}{$\%$} & \multicolumn{1}{l}{ No. } & $\%$ \\
Specific neurological or & & & & \\
psychiatric & 2782 & $69 \cdot 5$ & 2586 & $67 \cdot 4$ \\
Anatomical & 150 & 3.8 & 140 & $3 \cdot 7$ \\
Non-neurological & 55 & $1 \cdot 4$ & 48 & $1 \cdot 2$ \\
Undiagnosed & 1013 & $25 \cdot 3$ & 1062 & $27 \cdot 7$ \\
Total & 4000 & & 3836 & \\
\hline
\end{tabular}

Table 2 Top 20 diagnoses 1977-1987

\begin{tabular}{lrl}
\hline & & Per cent \\
\hline No diagnosis & 2075 & 26.5 \\
Epilepsy & 813 & $10 \cdot 4$ \\
Tension headache & 589 & $7 \cdot 5$ \\
CVD & 576 & $7 \cdot 4$ \\
Migraine & 389 & $5 \cdot 0$ \\
Entrapment neuropathy & 345 & $4 \cdot 4$ \\
Conversion hysteria & 297 & $3 \cdot 8$ \\
Anatomical & 290 & 3.7 \\
MS & 274 & $3 \cdot 5$ \\
Vaso-vagal & 163 & $2 \cdot 1$ \\
Hyperventilation & 159 & $2 \cdot 0$ \\
Parkinson's disease & 149 & 1.9 \\
Post-traumatic syndrome & 139 & $1 \cdot 8$ \\
Dementia & 121 & $1 \cdot 5$ \\
Peripheral neuropathy & 107 & 1.4 \\
Depression & 106 & 1.4 \\
Non-neurological & 103 & $1 \cdot 3$ \\
Cervical radiculopathy/myelopathy & 94 & 1.2 \\
Lumbar spondylosis & 77 & 1.0 \\
Essential tremor & 74 & 0.9 \\
\hline
\end{tabular}

effect on my own case material of whether or not a junior member of staff was seeing new outpatient referrals on my behalf. The analysis suggested that this factor did not influence the pattern of case distribution. Other factors have exerted an effect. The establishment of the Princess Margaret Migraine Clinic at Charing Cross Hospital has led to a substantial decline in the number of migraine patients seen by the author in his own clinic, but has had no effect on the number seen at Hillingdon Hospital.

\section{Discussion}

In one of the first attempts to determine case distribution in neurological practice, 72 American neurologists provided data on 20 successive cases, whether new or follow-up, seen at either outpatient or inpatient consultation. ${ }^{5}$ Standard diagnostic criteria were not used. The four commonest diagnoses were headache, convulsive disorders, vascular disease and psychiatric disorders, representing $52 \%$ of the total. The corresponding figure from my own data (though the categories are not immediately comparable) is $46 \%$. An ambitious study, surveying 10372 doctors in the United States, provided information on the case mix seen by a variety of specialists. ${ }^{6}$ Over a 3 day period, physicians recorded all their patient encounters including ward cases and both new and old outpatients. Using the eighth revision of the International Classification of Diseases the 20 most common problems or diagnoses were tabulated for each medical specialty. Certain conditions were seen with a similar frequency to that found in the present study (epilepsy $9.8 \%$, multiple sclerosis $3.6 \%$ ) but others (no diagnosis $9.8 \%$, headache or migraine, $5.7 \%$ ) less often. A survey more immediately comparable to the present one reviewed the diagnoses in 624 consecutive neurological consultations. ${ }^{7}$ The leading five diagnoses or symptoms were headache $(18.6 \%)$, epilepsy $(12.4 \%)$. "functional" $(11.0 \%)$, cerebrovascular $(8 \cdot 4 \%)$ and disc disease $(5 \cdot 0 \%)$.

Clearly, case distribution can differ from institute to institute, both for inpatient material ${ }^{8}$ and for outpatients. ${ }^{6}$ Furthermore, in areas, like London, where patients can readily seek further opinions, a substantial proportion of patients may have previously sought a neurological opinion elsewhere. In my first survey,' approximately a quarter of the patients with Parkinson's disease, multiple sclerosis and epilepsy had been seen previously by another neurologist. Furthermore, particular clinical interests, and, perhaps, a corresponding lack of expertise in other areas, are likely to influence the distribution of disease as reported by an individual neurologist. Despite these reservations, an analysis of a sufficient number of outpatient consultations provides invaluable information on the practice of neurology with implications for health care and for teaching.

\section{References}

1 Perkin GD. Pattern of neurological outpatient practice: implications for undergraduate and postgraduate training. J R S Med 1986;79:655-7.

2 Vahlquist B. Migraine in children. International Arch Allergy and Applied Immunology 1955;7:348-55.

3 McDonald WI, Halliday AM. Diagnosis and classification of multiple sclerosis. Br Med Bull 1977;33:4-8.

4 Guze SB. The diagnosis of hysteria: what are we trying to do. Am J Psychiatry 1967;124:77-84.

5 Rose AS. Graduate training in neurology. An assessment based on the opinions of 80 neurologists in private practice. Arch Neurol 1971;24:165-8.

6 Mendenhall RC. Medical Practice in the United States. A Special Report of the Robert Wood Johnson Foundation. Princeton, New Jersey. 1981.

7 Murray TJ. Concepts in undergraduate neurological teaching. Clin Neurol Neurosurg 1977;79:273-84.

8 Guez M, Anderson PJ. Frequency of neurologic diagnoses: implications for curriculum design. $M$ Sinai $J$ Med 1980;47:364-72. 